always has to remain so" (pp. 48/49). This is perceived to be an intellectual prison of our own choosing.

She deals with the subjects of international jurisdiction and its exceptions and then moves on to human rights and points out the specialty of this law, the obligations owed directly to individuals. She explains the various views on human rights and states that she prefers "the position that human rights are demands of a particular intensity made by individuals vis-à-vis their governments" (p. 105). It is up to everyone of us to participate in this fight for human rights, because international human rights bodies cannot succeed alone. The 1993 Vienna World Conference for Human Rights could have helped, but "states showed no interest" (p. 110).

A whole separate chapter is reserved for the particular human right of self-determination, where she analyses the UN Charter, GA Res. 1514 (XV) and 1541 (XV), and the Helsinki Final Act, as well as GA Res. 2625 (XXV) when concerned with self-determination beyond colonialism.

Regarding minorities in a time when states have begun to disintegrate, she asks who has the right to self-determination, how it is limited by the requirement of territorial integrity, whether it is limited to the exercise of political rights and she concludes that "(...) the right of self-determination continues beyond the moment of decolonization, and allows choices as to political and economic systems within the existing boundaries of the state" (p. 123), yet, as such, minorities do not have such a right. "It is today fashionable among political leaders to invoke the right of self-determination" (p. 124) and aid minorities, but to the international lawyer the rhetoric is - at best - confused. Rosalyn Higgins reminds the reader of the imminent danger of allowing it to be "all things to all men".

The work also contains detailed analyses on notion and courts, equity and proportionality etc., which are all well worth reading and enjoying.

The whole book is courageous, refreshing, outstanding. Do not miss it!

Dagmar Reimmann

\title{
Ernst-Otto Czempiel
}

Die Reform der UNO - Möglichkeiten und Mißverständnisse

BsR, C.H. Beck Verlag, München, 1994, 199 S., DM 19,80

Das Buch des Frankfurter Politikwissenschaftlers E.-O. Czempiel ist eine Publikation der Hessischen Stiftung Friedens- und Konfliktforschung.

Unterteilt in acht Kapitel befaßt es sich mit den Erkenntnissen der Theorie, den Erfahrungen der Vergangenheit und - in der Hauptsache - den aktuellen Problemen, Ideen und konkreten Lösungsvorschlägen, insbesondere der Erweiterung des Sicherheitsrates, der Friedenserzwingung, dem Ausbau des Peacekeeping, der Verstärkung der Friedensschaf- 
fung, der Vorbeugung von Konflikten und der unbedingt nötigen Reform der Außenpolitik der einzelnen Mitgliedstaaten der UNO.

Dieses Werk richtet sich jedoch nicht, wie bei diesen Themen anzunehmen, an die Wissenschaft, will nicht deren Forschung über die UNO voranbringen. Vielmehr richtet es sich an Funktionsträger in Politik und Gesellschaft, informiert darüber, was die Organisation leisten kann und was nicht, will den Mythos beseitigen, die Diskussion beleben. Und kann damit vielleicht doch Wissenschaftler ansprechen und den Elfenbeinturm der Wissenschaft aufschließen, denn diese müßte sich mehr um die Bedürfnisse dieser UNOPolitik kümmern.

Die Arbeit Czempiels ist auch kein Lehrbuch über die Organisation der UNO und ihre Sonderorganisationen; sie bietet vielmehr eine Funktionsanalyse der UNO, eine sehr kritische Betrachtung des Leistungsvermögens der UNO im internationalen System.

Den 'Neuanfang' der UNO ab 1990, die Renaissance der Organisation, sieht Czempiel unter dem Stichwort des Arbeitsprinzips 'Kooperation' realistisch bis positiv. "Was diese Zusammenarbeit ausrichten kann, wird gelegentlich übertrieben dargestellt. Weder sind die Vereinten Nationen eine Weltregierung, noch sind sie der Autor und der Kontrolleur jener neuen Weltordnung, deren Umrisse so undeutlich geblieben sind wie der Begriff selbst. (...) Die Gründungsväter von 1945 wollten mit den VN das 'Rathaus der Welt' (Vandenberg) errichten, in dem die Staaten ihre Konflikte nicht mit Gewalt, sondern mit Kooperation und Kompromiß lösen können. Weil dieser Ansatz nicht nur vielversprechend, sondern auch konzeptuell plausibel war, kehrte er sofort wieder, nachdem der OstWest-Konflikt (...) zu Ende gegangen war. Warum sollte es jetzt nicht möglich sein, die Probleme der Welt kooperativ anzugehen?" (S. 17). Vielleicht könnte die UNO wenigstens zum Weltpolizisten werden, wenn schon nicht zur Weltregierung, und sind nicht das Kriegsverbrechertribunal für Jugoslawien, der UNHCR, die Arbeitsgruppe für die Zukunft der Vereinten Nationen positive Signale?

Trotzdem wird die UNO gestoppt, mindestens gebremst durch Haushaltsprobleme und ist überfordert mit der Eliminierung des Krieges. Viel hat sich verändert. Kapitel VII-Einsätze, Somalia, Bosnien sind neu. Früher beschränkt durch Art. 2 (7) der Charta, eröffnen sich jetzt neue Möglichkeiten.

Czempiels Ansicht nach ist z.B. das Peacekeeping ein 'Volltreffer', und wenn sich die UNO auf ihre in der Charta enthaltenen Möglichkeiten, der Gewaltanwendung vorzubeugen, besinnen könnte, wäre der wichtigste Schritt getan. "Für diese Reform der Funktion brauchte die Charta der Vereinten Nationen nicht geändert zu werden, sie müßte nur anders interpretiert werden. Geändert werden muß die Außenpolitik der UN-Mitglieder. Sie behandeln die Vereinten Nationen wie jede andere internationale Organisation, wie einen beliebigen multinationalen Kontext. Das bietet ihnen die Möglichkeit, ein schizophrenes Verhalten in der Außenpolitik an den Tag zu legen, in den Vereinten Nationen deren Normen und Verhaltensvorschriften deklaratorisch zu akzeptieren und zu begrüßen, in ihrer Außenpolitik darauf aber keine Rücksicht zu nehmen" (S. 23). Sinnvollerweise sollte eine auf umfassende Sicherheit ausgerichtete Politik die Herrschaftssysteme der 
Mitglieder demokratisieren und ihre Mitarbeit in einer internationalen Organisation institutionalisieren.

Weiterhin kritisiert der Autor den Begriff 'Staat', den die UNO anwendet, und plädiert für ein neues Konzept. Seiner Ansicht nach kann man mit dem herkömmlichen Begriff nicht mehr operieren. "Die Vereinten Nationen müssen sich (...) soweit reformieren, daß sie ein angemessenes Staatenbild verwenden und daraus auch Konsequenzen für die eigene Organisation ziehen können. (...) In der Gesellschaftswelt liegt die Souveränität nicht bei der Exekutive, sondern bei der Gesellschaft und ihrer politischen Repräsentanz, der Legislative" (S. 45).

Zur 'Agenda für den Frieden' (1992) des Generalsekretärs äußert er sich wie folgt: "Deren Empf ehlungen politisch zu verwirklichen, ist natürlich nicht nur den Vereinten Nationen, sondern in erster Linie ihren Mitgliedsstaaten aufgegeben. Aber auch die politische Praxis der Vereinten Nationen besitzt jetzt einen Kompaß, der sich nicht mehr am Ausbruch der Gewalt, sondern an deren Verhinderung orientiert und der die Ursachen der Aggression nicht immer nur in bösen Absichten, sondern auch in wirtschaftlicher Not, sozialer Ungerechtigkeit und politischer Unterdrückung lokalisiert. Zumindest programmatisch haben die Vereinten Nationen damit einen großen Schritt in die richtige Richtung getan" (S. 46). Wie die Praxis aussehen wird, ist ungewiß.

In seiner Analyse des Sicherheitsrates zeigt er die Vergangenheit, insbesondere die Blockade durch das Veto auf und befaßt sich dann mit der vieldiskutierten Erweiterung dieses Organs. Zum Thema der Zusammensetzung des Sicherheitsrates zitiert er: "Die Zahl der Ständigen Sicherheitsrats-Mitglieder zu erhöhen, wäre keinesfalls gleichbedeutend damit, auch die Kompetenzen des Rates zu verbessern." ${ }^{1}$ Czempiel selbst meint, daß die Reformierung der Praxis der UNO und ihre Ausrichtung auf die neue Bedingungen so wichtig seien, daß die Bereitschaft, "die kostbare Zeit mit einer Diskussion um die Änderung der Charta zu vergeuden" (S. 60), nicht sehr groß sei. Außer den USA und den Niederlanden habe auch niemand ein wirkliches Interesse an einem Ständigen Sitz für Deutschland und Japan, Afrika und Lateinamerika ginge es um mehr echte Gerechtigkeit bei der Sitzverteilung.

In seiner Besprechung der Friedenserzwingung geht er detailliert auf die relevanten Vorschriften der Charta ein und rät zur Vorsicht. "Weil der Sicherheitsrat das Monopol der militärischen Gewaltanwendung besitzt, die allen anderen Staaten versagt bleibt, müssen die Anwendungsregeln des Kapitels VII strikt befolgt werden. Nur sie konstituieren jene Legitimität der internationalen Organisation, die den Säbel des Imperialismus in das Schwert der internationalen Gerechtigkeit umwandelt" (S. 69). In bezug auf Artikel 2 (4) betont er, daß rechtliche Zulässigkeit niemals mit politischer Richtigkeit verwechselt werden dürfe. Am Beispiel des Golfkriegs zeigt Czempiel, daß Effizienz allein kein Beurteilungskriterium sein kann, der Zweck heilige keinesfalls die Mittel - "Der Sicherheits- 
rat wurde ein-, aus- und wieder zugeschaltet, je nach Gründen der Opportunität" (S. 98) und gibt an, wie seiner Meinung nach ein Eingreifen der UNO nach den Vorschriften der Charta hätte aussehen müssen. "Den Sicherheitsrat zu reformieren heißt daher, weitere Umgehungen seiner Verfahrensbestimmungen randscharf zu unterbinden" (S. 73). Die Bestimmungen des Kapitel VII müßten erst einmal verwirklicht werden, bevor man über eine Reform entscheiden kann, was jedoch Generalsekretär Boutros Boutros-Ghali zur Zeit für unrealistisch hält. Als wichtigstes Kriterium für jegliche Aktionen sieht der Autor den Konsens an.

Er äußert sich zu Interventionen in Bürgerkriege, zum Ausbau des Peacekeeping, wobei er die Friedenssicherung an regionale Unterorganisationen der UNO abgeben will, der Sicherheitsrat jedoch die Oberaufsicht führen soll, und gibt dafür erfolgreiche und erfolglose Operationen als Beispiel an. Die UNO-Maßnahmen setzten generell zu spät ein, nämlich wenn bereits Gewalt angewendet wurde, wenn das jeweilige Herrschaftsverhältnis zwischen politischem System und gesellschaftlicher Umwelt gescheitert ist. Vorbeugung ist gefragt!

"Die Ständigen Mitglieder" des Sicherheitsrates (...) und alle Staaten fürchten natürlich eine detaillierte Verpflichtung zur friedlichen Streitbeilegung (...), die politischen Systeme (...) sind nicht daran interessiert, daß die Verfahren der friedlichen Streitbeilegung weiterentwickelt und implementiert werden. Sie sehen darin eine Einschränkung ihres Herrschaftsanspruchs nach innen und ihres Machtanspruchs nach außen" (S. 141). Um dieses Problem zu überwinden, muß die UNO ein neues, eingeschränktes Souveränitätsverständnis entwickeln und Wege finden, den Widerstand der Regierungen zu überwinden, die in jeder Aktion den Präzedenzfall sehen. Hier hat der Generalsekretär in seiner 'Agenda für den Frieden' schon den ersten Schritt unternommen.

Der Autor zeigt vier Schritte zur Vorbeugung: Unerläßlich sind die Parlamentarisierung der UNO, Abrüstung und Einstellung des Waffenhandels, Demokratisierung der Herrschaftssysteme, die Früherkennung von Konflikten. Hierbei wird das konsequente Ausnutzen von UN-Präsenz verlangt.

Am Ende kehrt Czempiel zu seinem Anfang zurück, zur Reform der Außenpolitik. Sein Rat: "(...) am Konzept von Außenpolitik muß ansetzen, wer die Vereinten Nationen reformieren will. Denn nicht die Organisation muß reformiert werden, sondern das Denken über sie. Sie muß hervorgeholt werden aus der Nische, in der die Politiker und Diplomaten die eher ornamentalen Veranstaltungen aufbewahren, die symbolischen Akte, die Emporen glänzender Rhetorik, die Erklärungen der guten Absichten. Die internationale Organisation muß endlich begriffen werden als eine der wichtigsten operativen Strategien, die der Gegenwart zur Verfügung stehen und von den post-modernen Staaten des Westens als Höchstzweck rational benutzt werden sollten. Nichts wäre falscher, als die Reform der Vereinten Nationen nur unter dem Aspekt der Erweiterung des Sicherheitsrates oder der Verschlankung der Sekretariatsbürokratie zu diskutieren (...)" (S. 181). Was als 'Reform der Vereinten Nationen' bezeichnet wird, muß also ganz wörtlich verstanden werden: "Die Nationalstaaten müssen sich selbst reformieren, damit ihre Außen- 
politik reformiert wird, sich abwendet von der Anwendung eigener Macht und sie umwandelt in fremde Pflichten. Das Medium dazu ist die internationale Organisation" (S. 182).

Die Arbeit Czempiels ist von außerordentlicher Qualität. Ich kann mich dem Autor nur anschließen: "Dieses Buch hätte längst geschrieben werden müssen" (S. 7).

Dagmar Reimmann

Wolfgang Schmale (Hrsg.)

Human Rights and Cultural Diversity

Keip Publishing, Goldbach, 1993, 348 S.

$\mathrm{Zu}$ diesem Buch greift man schon aufgrund des Titels mit sehr hohen Erwartungen. Sie werden noch gesteigert durch die einleitende Feststellung, den Autoren sei es gelungen, einen "völlig neuen und kritischen Aspekt herauszuarbeiten" (S. VII). Daß diese Erwartungen und dieser Anspruch nur teilweise erfüllt werden, mag daran liegen, daß die elf Teilnehmer einer vom Herausgeber im Februar 1992 an der Ruhr-Universität in Bochum durchgeführten Konferenz, deren dreizehn Beiträge hier vorgelegt werden, dort offenbar zum Teil aneinander vorbeigeredet haben müssen. Anders sind - auch bei einer "kontroversen Diskussion" (S. VII) - manche Diskrepanzen nicht zu erklären.

Im drei Beiträge umfassenden Grundsatzteil gibt zunächst der Herausgeber Wolfgang Schmale (S. 3-27) einen Überblick über die Themenstellung der übrigen Referate und stellt dann zu Recht kritisch fest, daß die Geschichte der Menschenrechte bisher fast ausschließlich als Ideengeschichte behandelt worden sei, unter Vernachlässigung des gesellschaftlichen Prozesses ihrer Durchsetzung (S. 19, 21). Jörn Rüse behandelt die Menschenrechte aus der Perspektive der Universalgeschichte (S. 28-46) und geht dabei ausführlich der Frage nach, ob die Propagierung der für die europäische Geistesgeschichte spezifischen Menschenrechtsvorstellungen nicht andere Kulturen ihrer Identität beraube. Unter Hinweis darauf, daß die Eigenbezeichnungen vieler Völker schlechthin "Mensch" bedeuten, stellt er andererseits eine auf Universalisierung zielende Entwicklungsdynamik fest und ruft schließlich zu interkultureller Toleranz auf (S. 35, 42, 44).

Wirklich aufregend aber wird es in dem folgenden Beitrag von Harro v. Senger über zwei Perioden der Menschenrechte, nämlich die einer beschränkten Konzeption vor der VNMenschenrechtserklärung von 1948 und der einer erst von daher datierenden wirklich universalen (S. 47-100). Der Verfasser behandelt nämlich nicht, was unter Menschenrechten zu verstehen sei, sondern die bisher kaum gestellte Frage, wem diese Rechte denn zustehen sollen. Er kommt dabei zu dem zunächst überraschenden Ergebnis, daß der Begriff "Mensch" von den Denkern der "patriarchalischen europäischen Aufklärung" nicht 